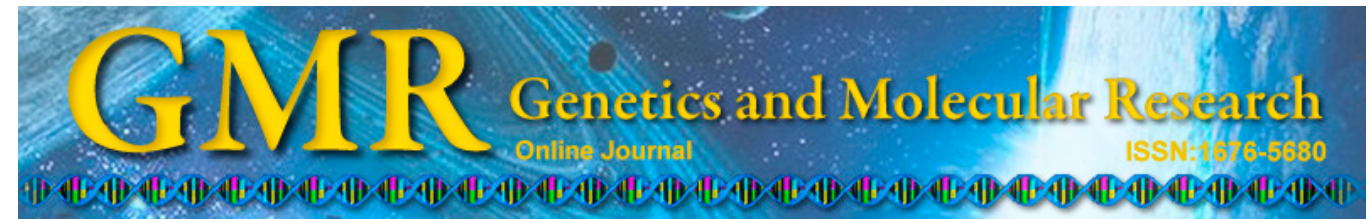

\title{
Functional analysis of differentially expressed genes associated with glaucoma from DNA microarray data
}

\author{
Y. Wu' ${ }^{1}$ W.D. Zang ${ }^{2}$ and W. Jiang ${ }^{1}$ \\ ${ }^{1}$ Department of Ophthalmology, Chengdu Military General Hospital, \\ Chengdu, China \\ ${ }^{2}$ Department of Biology, Shanghai Jiaotong University, Shanghai, China \\ Corresponding author: W. Jiang \\ E-mail: jiangweicdh@hotmail.com
}

Genet. Mol. Res. 13 (4): 9421-9428 (2014)

Received August 29, 2013

Accepted May 2, 2014

Published November 11, 2014

DOI http://dx.doi.org/10.4238/2014.November.11.7

\begin{abstract}
Microarray data of astrocytes extracted from the optic nerves of donors with and without glaucoma were analyzed to screen for differentially expressed genes (DEGs). Functional exploration with bioinformatic tools was then used to understand the roles of the identified DEGs in glaucoma. Microarray data were downloaded from the Gene Expression Omnibus (GEO) database, which contains 13 astrocyte samples, 6 from healthy subjects and 7 from patients suffering from glaucoma. Data were pre-processed, and DEGs were screened out using $R$ software packages. Interactions between DEGs were identified, and networks were built using Search Tool for the Retrieval of Interacting Genes/Proteins (STRING). GENECODIS was utilized for the functional analysis of the DEGs, and GOTM was used for module division, for which functional annotation was conducted with the Database for Annotation, Visualization, and Integrated Discovery (DAVID). A total of 371 DEGs were identified between glaucoma-associated samples and normal samples. Three modules included in the PPID database were generated with 11,12 , and 2 significant functional annotations, including immune system processes, inflammatory responses, and synaptic
\end{abstract}


vesicle endocytosis, respectively. We found that the most significantly enriched functions for each module were associated with immune function. Several genes that play interesting roles in the development of glaucoma are described; these genes may be potential biomarkers for glaucoma diagnosis or treatment.

Key words: Astrocytes; Optic nerve; Differentially expressed genes; Module analysis; Functional analysis

\section{INTRODUCTION}

Glaucoma is the second leading cause of irreversible blindness worldwide. There are approximately 10 million glaucoma patients in China, accounting for $5.3-21 \%$ of all blind people (Quigley and Broman, 2006). Glaucoma is characterized by depressed optic nerve atrophy and visual field defects. Increased intraocular pressure is a major risk factor of glaucoma (Morrison et al., 2005). According to surveys, 50\% of patients do not know they are suffering from glaucoma in developed countries, while this value reaches up to $90 \%$ in developing countries (Quigley and Broman, 2006). Once late-stage disease develops, it is irreparable.

Many studies have been performed to uncover the pathogenesis of glaucoma, and some progress has been achieved. Immune function is thought to play an important role. Tezel et al. (1998) found that circulating antibodies against retinal small heat-shock proteins may have pathogenic significance in some glaucoma patients. Ikeda et al. (2002) found a similar role for serum antibody against neuron-specific enolase, and further confirmed its clinical significance. Fraenkl et al. (2011) investigated plasma citrate levels as a potential biomarker for glaucoma. In addition, the relationship between glaucoma and inflammatory response has been investigated (Cohen et al., 1996; Zemba et al., 2002; Bodh et al., 2011; Siasos et al., 2011). Proteomic technologies have been used to delineate the process and to identify potential biomarker proteins (Wong et al., 2011; Tezel et al., 2012).

As glaucoma pathogenesis is rather complicated, omic technologies are necessary to fully understand the disease. Microarray technology is a widely used tool for exploring the mechanism from the perspective of gene expression profiles. Therefore, we compared astrocyte samples from optic nerves of donors with and without glaucoma by using microarray technology to identify key genes that may be useful as drug targets.

\section{MATERIAL AND METHODS}

The microarray data set GSE2378 (Kompass et al., 2008) was downloaded from the Gene Expression Omnibus (GEO) database, which includes 13 astrocyte samples, 6 from healthy subjects and 7 from glaucoma patients. Data were collected using the platform GPL8300 [HG_U95Av2] Affymetrix Human Genome U95 Version 2 Array. Both the annotation files provided by Affymetrix and the raw data were obtained for follow-up analysis.

Data were pro-processed using the Affy package (Gautier et al., 2004) of $R$. Differential expression analysis was performed using the Limma package (Smyth, 2005). A falsediscovery rate $(\mathrm{FDR})<0.05$ and a $|\log \mathrm{FC}|>1$ (FC, fold-change) were set as cut-off values to screen differentially expressed genes (DEGs).

Because genes (or proteins) usually work together to exert biological functions, build- 
ing interaction networks is beneficial for interpreting their functional roles. The online software Search Tool for the Retrieval of Interacting Genes/Proteins (STRING) (Szklarczyk et al., 2011) was used to examine interactions among DEGs and to establish the network. STRING scores for each interaction were based on actual experimental data and data mining from the literature. Interactions with a STRING score $>0.8$ were selected for subsequent analysis.

Pathway-enrichment analysis was carried out for DEGs in the network with GENECODIS (Lodish, 2008). The cut-off was set as an adjusted (adj) $\mathrm{P}$ value $<0.05$. GENECODIS is a web-based tool designed for functional analysis and integrates different information resources (GO, KEGG, and SwissProt) for gene annotation.

It is generally considered that genes (or proteins) with similar functions are prone to gather in the same module (Herrgard et al., 2006). Therefore, module analysis is useful for examining biological processes. Gene Ontology Tree Machine (GOTM) (Li et al., 2012) combined with the protein-protein interaction database (PPID) was utilized for module-enrichment analysis based on the hypergeometric distribution arithmetic. The cut-off value was set as adj $\mathrm{P}<0.05$.

Database for Annotation, Visualization, and Integrated Discovery (DAVID) (Dennis Jr. et al., 2003) was then used to identify the functions of the DEGs contained in the modules. The cut-off value was set as adj $\mathrm{P}<0.05$.

\section{RESULTS}

\section{DEGs}

The microarray data was pre-processed and normalized (Figure 1). Differential analysis identified a total of 371 DEGs according to the criteria described above $(\mathrm{P}<0.05$ and $|\log \mathrm{FC}|>1)$, of which 130 genes were up-regulated and 214 genes were down-regulated.

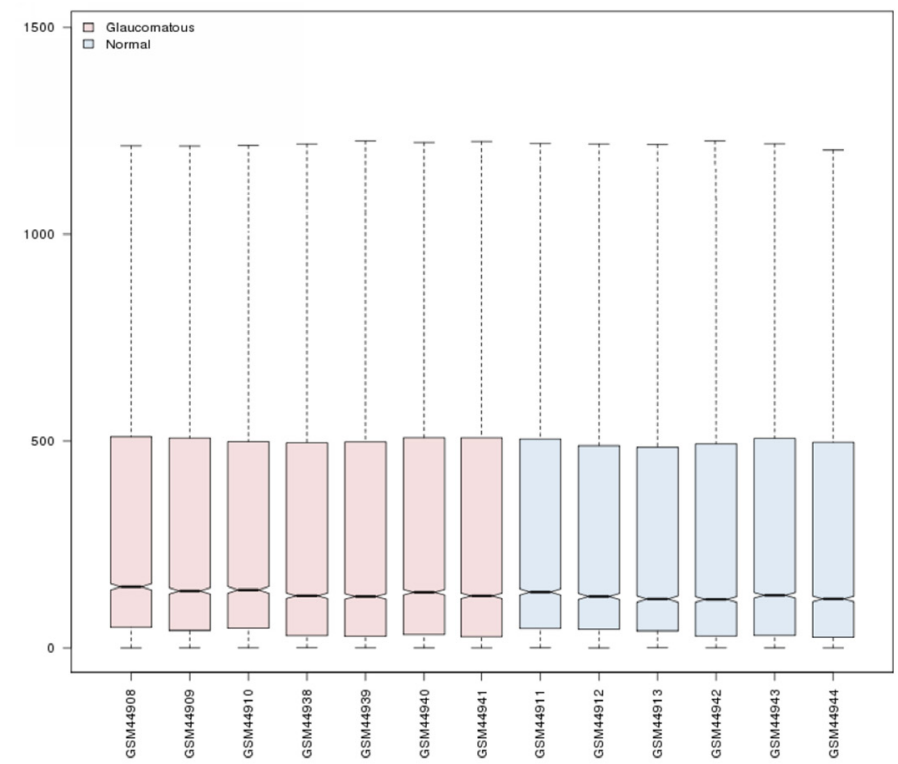

Figure 1. Box plot for normalized gene expression data, 6 blue boxes for samples from normal subjects and 7 pink boxes for samples from glaucoma patients. Black lines in the boxes represent the medians. The medians are nearly on the same line, indicating good performance of our normalization method. 


\section{Interaction network and functional analysis}

A total of 308 pairs of interactions were retrieved with high confidence using STRING (Table 1). Twelve significant pathways (Table 2) were obtained using GENECODI, among which the GO 000716 enzyme-linked receptor protein signaling pathway was the most significant.

Table 1. Functional annotations for the three modules.

\begin{tabular}{|c|c|c|}
\hline GO term & adj $P$ value & Description \\
\hline \multicolumn{3}{|l|}{ A. } \\
\hline 2376 & $5.96 \mathrm{E}-04$ & Immune system process \\
\hline 6950 & $3.85 \mathrm{E}-03$ & Response to stress \\
\hline 7165 & $4.95 \mathrm{E}-03$ & Signal transduction \\
\hline 23052 & $5.55 \mathrm{E}-03$ & Signaling \\
\hline 23060 & $6.31 \mathrm{E}-03$ & Signal transmission \\
\hline 23046 & $6.31 \mathrm{E}-03$ & Signaling process \\
\hline 50896 & $7.82 \mathrm{E}-03$ & Response to stimulus \\
\hline 50789 & $1.35 \mathrm{E}-02$ & Regulation of biological process \\
\hline 65007 & $1.74 \mathrm{E}-02$ & Biological regulation \\
\hline 32501 & $3.43 \mathrm{E}-02$ & Multicellular organismal process \\
\hline 50794 & $3.74 \mathrm{E}-02$ & Regulation of cellular process \\
\hline \multicolumn{3}{|l|}{ B. } \\
\hline 6954 & 4.03E-07 & Inflammatory response \\
\hline 9611 & 8.63E-06 & Response to wounding \\
\hline 6952 & $1.47 \mathrm{E}-05$ & Defense response \\
\hline 40011 & 4.65E-05 & Locomotion \\
\hline 7166 & $5.82 \mathrm{E}-05$ & Cell surface receptor-linked signaling pathway \\
\hline 7186 & $6.50 \mathrm{E}-05$ & G-protein-coupled receptor protein signaling pathway \\
\hline 2376 & $1.15 \mathrm{E}-04$ & Immune system process \\
\hline 23033 & $1.00 \mathrm{E}-03$ & Signaling pathway \\
\hline 42221 & $1.06 \mathrm{E}-03$ & Response to chemical stimulus \\
\hline 23052 & $1.34 \mathrm{E}-03$ & Signaling \\
\hline 6950 & $2.61 \mathrm{E}-03$ & Response to stress \\
\hline 50896 & $1.76 \mathrm{E}-02$ & Response to stimulus \\
\hline \multicolumn{3}{|l|}{ C. } \\
\hline 48488 & $2.10 \mathrm{E}-03$ & Synaptic vesicle endocytosis \\
\hline 48489 & $7.85 \mathrm{E}-03$ & Synaptic vesicle transport \\
\hline
\end{tabular}

adj $=$ adjusted.

Table 2. Functional-enrichment analysis results for DEGs in the network.

\begin{tabular}{llr}
\hline Term & Function & FDR \\
\hline GO:0007167 & Enzyme-linked receptor protein signaling pathway & $3.95 \mathrm{E}-06$ \\
GO:0042127 & Regulation of cell proliferation & $4.48 \mathrm{E}-05$ \\
GO:0007178 & Transmembrane receptor protein serine/threonine kinase signaling pathway & $2.18 \mathrm{E}-04$ \\
GO:0007179 & Transforming growth factor beta receptor signaling pathway & 0.0021 \\
GO:0007155 & Cell adhesion & 0.002219 \\
GO:0022610 & Biological adhesion & 0.002272 \\
GO:0001501 & Skeletal system development & 0.003348 \\
GO:0040007 & Growth & 0.01171 \\
GO:0007166 & Cell surface receptor-linked signal transduction \\
GO:0042060 & Wound healing & 0.01522 \\
GO:0008284 & Positive regulation of cell proliferation & 0.02018 \\
GO:0006952 & Defense response & 0.027672 \\
\hline
\end{tabular}

$\mathrm{FDR}=$ false-discovery rate.

\section{Module analysis}

Three significant modules (Figure 2) were established for the DEGs using GOTM. 
Eleven, 12, and 2 functional annotations were revealed by DAVID for each module (Table 1), with the most enriched including immune system processes, inflammatory responses, and synaptic vesicle endocytosis, respectively. Considering that the latter two processes are closely associated with immunity, the immune function may play a critical role in the pathogenesis of glaucoma.
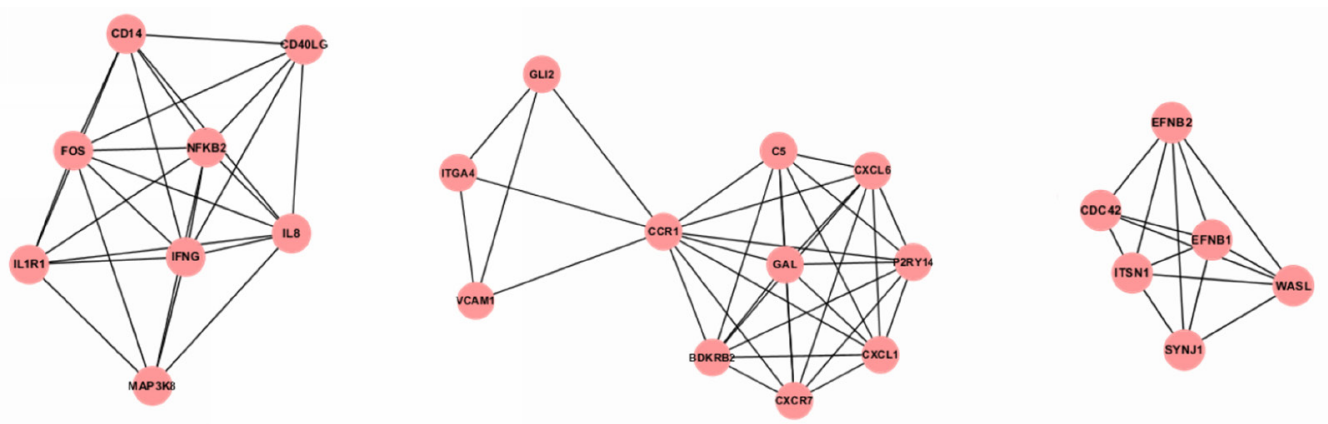

Figure 2. Three modules for all DEGs involved in the network.

\section{DISCUSSION}

Glaucoma is a degenerative disease that results from aberrant immune responses. Since it causes serious damage to the eyes, early diagnosis and proper treatment is particularly important. In this study, chip data for astrocytes from donors with and without glaucoma were analyzed using bioinformatic tools to identify critical genes and significant biological functions. A total of 371 DEGs were identified, and an interaction network was generated. Functional analysis revealed 12 significantly enriched pathways. Module analysis was conducted to delineate their biological functions, and immune function was found to be important in glaucoma pathogenesis.

The highly significant pathways included enzyme-linked receptor protein signaling pathways, regulation of cell proliferation, and the transmembrane receptor protein serine/threonine kinase signaling pathway. The cell surface receptor-linked signal transduction pathway showed the maximum number of DEGs. Four pathways identified were associated with signal transduction (Table 2). In addition, defense response, wound healing, and regulation of cell proliferation were also identified. These pathways were found to be associated with glaucoma, suggesting the potential biological significance of these DEGs.

Module analysis was carried out to further describe these genes. Three modules were obtained with various biological function patterns. The first module consisted of 11 DEGs; the most significant annotation was the immune system process. The second module included 12 DEGs associated with the inflammatory response and response to stress. The last module contained only 2 DEGs, and was related to synaptic vesicle endocytosis.

Previous studies have shown that the immune system can simultaneously confer a neuroprotective effect and neuronal injury (Tezel and Wax, 2004). The balance between these 2 opposing effects is regulated by glial cells; therefore, astrocytes were examined in this study. Several DEGs, including interleukin-1 receptor, type I (ILIRI), interleukin 8 (IL-8), CD40 
ligand, interferon gamma (IFNG), nuclear factor of kappa light polypeptide gene enhancer in B-cell 2, and CD14, are involved in immune system processes. While IFNG is up-regulated, the others are down-regulated in glaucoma. IFNG is a potent activator of macrophages. Macrophages are implicated in antigen presentation, and thus regulate down-stream responses. The other gene worthy of note is purinergic receptor P2Y, G-protein-coupled 14 (P2RY14). P2RY14 plays a role in extending known immune functions of P2Y receptors (Arase et al., 2009) and neuroimmune function. Mochizuki et al. (2012) found that oxidative stress significantly stabilizes IL-8 mRNAs in human trabecular meshwork cells. Similar conclusions have been obtained in other studies (Malvitte et al., 2007; Luna et al., 2009). However, we observed that $I L-8$ was down-regulated in astrocytes. Therefore, there may be different patterns of immune responses in various cells, indicating the roles of various cell types. The identification of key genes involved in immune function is important as these may be used as targets of immunomodulation for treating glaucoma (Tezel, 2013). Therefore, these DEGs are promising candidates for treatment options.

The stimulation of oxidative stress on the immune response has been investigated previously (Tezel et al., 2007; Tezel, 2009, 2011; Pinazo-Durán et al., 2012). A study by Tezel et al. (2007) found that reactive oxygen species regulate the immune response by stimulating the antigen-presenting ability of glial cells and functioning as co-stimulatory molecules for antigen presentation. In our study, we identified several DEGs associated with the response to stress and the inflammatory response, including chemokine (C-X-C motif) ligand 1 (CXCL1), vascular cell adhesion molecule 1, chemokine (C-C motif) receptor 1 (CCRI), complement component 5 (C5), bradykinin receptor B2, chemokine (C-X-C motif) ligand 6 (CXCL6), and galanin $(G A L) . C C R 1$ is up-regulated in glaucoma according to our analysis, and its protein product is a member of the beta chemokine receptor family. This protein binds to ligands such as macrophage inflammatory protein 1 alpha (MIP-1 alpha), and is regulated upon activation of normal T-expressed and secreted protein (RANTES), monocyte chemoattractant protein 3 (MCP-3), and myeloid progenitor inhibitory factor-1 (MPIF-1). Chemokines and their receptor-mediated signal transduction pathways are critical for recruiting effector immune cells to the site of inflammation. GAL is also up-regulated and its product, galanin, takes part in a variety of physiological functions, such as nociception, arousal/sleep regulation, cognition, and many aspects of neuroendocrine activities (Mechenthaler, 2008). Because of its role in regulating energy metabolism and production, GAL may be used as a biomarker for monitoring or restoring the function of optical neural functions. CXCL6 is a cytokine that induces the directed migration of monocytes and neutrophils (Yoshie et al., 2001) and was found to be modulated in glaucoma (Liton et al., 2006; Lukas et al., 2008). Paylakhi et al. (2011) further reported that CXCL6 was regulated by pituitary homeobox 2 in glaucoma. Since the interactions among oxidative stress, inflammatory response, and immune response have been demonstrated as the basis for glaucoma, our findings increase the understanding of how these pathways interact.

Moreover, several genes related to the immune response and inflammatory responses were identified, including ILIR1, CXCL1, and C5. Few studies have investigated the involvement of these genes in the development of glaucoma. Additional studies using different techniques (Yan et al., 2009) are needed to further characterize their roles.

We systemically compared gene expression in astrocytes derived from optic nerves of donors with and without glaucoma and identified several DEGs associated with the stress response, immune response, and inflammatory response. Our analysis increases knowledge 
of the pathogenesis of glaucoma. The key genes implicated in the biological processes are potential targets for drug development.

\section{REFERENCES}

Arase T, Uchida H, Kajitani T, Ono M, et al. (2009). The UDP-glucose receptor P2RY14 triggers innate mucosal immunity in the female reproductive tract by inducing IL-8. J. Immunol. 182: 7074-7084.

Bodh SA, Kumar V, Raina UK, Ghosh B, et al. (2011). Inflammatory glaucoma. Oman. J. Ophthalmol. 4: 3-9.

Cohen RG, Wu HK and Schuman JS (1996). Glaucoma with inflammatory precipitates on the trabecular meshwork: a report of Grant's syndrome with ultrasound biomicroscopy of precipitates. J. Glaucoma 5: 266-270.

Dennis G Jr, Sherman BT, Hosack DA, Yang J, et al. (2003). DAVID: Database for Annotation, Visualization, and Integrated Discovery. Genome Biol. 4: 3.

Fraenkl SA, Muser J, Groell R, Reinhard G, et al. (2011). Plasma citrate levels as a potential biomarker for glaucoma. $J$. Ocul. Pharmacol. Ther. 27: 577-580.

Gautier L, Cope L, Bolstad BM and Irizarry RA (2004). Affy-analysis of Affymetrix GeneChip data at the probe level. Bioinformatics 20: 307-315.

Herrgard MJ, Lee BS, Portnoy V and Palsson BO (2006). Integrated analysis of regulatory and metabolic networks reveals novel regulatory mechanisms in Saccharomyces cerevisiae. Genome Res. 16: 627-635.

Ikeda Y, Maruyama I, Nakazawa M and Ohguro H (2002). Clinical significance of serum antibody against neuron-specific enolase in glaucoma patients. Jpn. J. Ophthalmol. 46: 13-17.

Kompass KS, Agapova OA, Li W, Kaufman PL, et al. (2008). Bioinformatic and statistical analysis of the optic nerve head in a primate model of ocular hypertension. BMC Neurosci. 9: 93.

Li Z, Cui G, Wang J, Yu Z, et al. (2012). Nemo-like kinase (NLK) involves in neuronal apoptosis after traumatic brain injury. Cell Mol. Neurobiol. 32: 381-389.

Liton PB, Luna C, Challa P, Epstein DL, et al. (2006). Genome-wide expression profile of human trabecular meshwork cultured cells, nonglaucomatous and primary open angle glaucoma tissue. Mol. Vis. 12: 774-790.

Lodish H (2008). Molecular Cell Biology. Macmillan Publishers, Ltd., London.

Lukas TJ, Miao H, Chen L, Riordan SM, et al. (2008). Susceptibility to glaucoma: differential comparison of the astrocyte transcriptome from glaucomatous African American and Caucasian American donors. Genome Biol. 9: R111.

Luna C, Li G, Liton PB, Qiu J, et al. (2009). Resveratrol prevents the expression of glaucoma markers induced by chronic oxidative stress in trabecular meshwork cells. Food Chem. Toxicol. 47: 198-204.

Malvitte L, Montange T, Vejux A, Baudouin C, et al. (2007). Measurement of inflammatory cytokines by multicytokine assay in tears of patients with glaucoma topically treated with chronic drugs. Br. J. Ophthalmol. 91: 29-32.

Mechenthaler I (2008). Galanin and the neuroendocrine axes. Cell Mol. Life Sci. 65: 1826-1835.

Mochizuki H, Murphy CJ, Brandt JD, Kiuchi Y, et al. (2012). Altered stability of mRNAs associated with glaucoma progression in human trabecular meshwork cells following oxidative stress. Invest. Ophthalmol. Vis. Sci. 53: 1734-1741.

Morrison JC, Johnson EC, Cepurna W and Jia L (2005). Understanding mechanisms of pressure-induced optic nerve damage. Prog. Retin. Eye Res. 24: 217-240.

Paylakhi SH, Fan JB, Mehrabian M, Sadeghizadeh M, et al. (2011). Effect of PITX2 knockdown on transcriptome of primary human trabecular meshwork cell cultures. Mol. Vis. 17: 1209-1221.

Pinazo-Durán MD, Zanón-Moreno V, García-Medina JJ and Gallego-Pinazo R (2013). Evaluation of presumptive biomarkers of oxidative stress, immune response and apoptosis in primary open-angle glaucoma. Curr. Opin. Pharmacol. 13: 98-107.

Quigley HA and Broman AT (2006). The number of people with glaucoma worldwide in 2010 and 2020. Br. J. Ophthalmol. 90: 262-267.

Siasos G, Tousoulis D, Siasou G, Moschos MM, et al. (2011). The association between glaucoma, vascular function and inflammatory process. Int. J. Cardiol. 146: 113-115.

Smyth GK (2005). Limma: Linear Models for Microarray Data, in Bioinformatics and Computational Biology Solutions Using R and Bioconductor. Springer, New York, 397-420.

Szklarczyk D, Franceschini A, Kuhn M, Simonovic M, et al. (2011). The STRING database in 2011: functional interaction networks of proteins, globally integrated and scored. Nucleic Acids Res. 39: D561-D568.

Tezel G (2009). The role of glia, mitochondria, and the immune system in glaucoma. Invest. Ophthalmol. Vis. Sci. 50: $1001-1012$

Tezel G (2011). The immune response in glaucoma: a perspective on the roles of oxidative stress. Exp. Eye Res. 93: 178-186.

Genetics and Molecular Research 13 (4): 9421-9428 (2014)

CFUNPEC-RP www.funpecrp.com.br 
Tezel G (2013). Immune regulation toward immunomodulation for neuroprotection in glaucoma. Curr. Opin. Pharmacol. 13: $23-31$.

Tezel G and Wax MB (2004). The immune system and glaucoma. Curr. Opin. Ophthalmol. 15: 80-84.

Tezel G, Seigel GM and Wax MB (1998). Autoantibodies to small heat shock proteins in glaucoma. Invest. Ophthalmol. Vis. Sci. 39: 2277-2287.

Tezel G, Yang X, Luo C, Peng Y, et al. (2007). Mechanisms of immune system activation in glaucoma: oxidative stressstimulated antigen presentation by the retina and optic nerve head glia. Invest Ophthalmol. Vis. Sci. 48: 705-714.

Tezel G, Yang X, Luo C, Cai J, et al. (2012). An astrocyte-specific proteomic approach to inflammatory responses in experimental rat glaucoma. Invest. Ophthalmol. Vis. Sci. 53: 4220-4233.

Wong TT, Zhou L, Li J, Tong L, et al. (2011). Proteomic profiling of inflammatory signaling molecules in the tears of patients on chronic glaucoma medication. Invest. Ophthalmol. Vis. Sci. 52: 7385-7391.

Yan L, Joachim SC, Pfeiffer N, Grus HF, et al. (2009). Analysis of antibody profiles against retinal antigens in serum from patients with glaucoma through mass spectrometry. Zhonghua Yan Ke Za Zhi 45: 625-630.

Yoshie O, Imai T and Nomiyama H (2001). Chemokines in immunity. Adv. Immunol. 78: 57-110.

Zemba M, Popescu M, Bobeico V, Bratulescu M, et al. (2002). Post-inflammatory glaucoma-methods of treatment. Oftalmologia 54: 17-22. 